\title{
Finite Approximate Controllability for Semilinear Heat Equations in Noncylindrical Domains
}

\author{
SILVANO B. DE MENEZES ${ }^{1}$, JUAN LIMACO ${ }^{2}$ and LUIS A. MEDEIROS $^{3}$ \\ ${ }^{1}$ Departamento de Matemática, Universidade Federal do Pará, \\ 60075-110 Belém, PA, Brasil \\ ${ }^{2}$ Instituto de Matemática, Universidade Federal Fluminense, \\ 24210-110 Niterói, Rio de Janeiro, Brasil \\ ${ }^{3}$ Instituto de Matemática, Universidade Federal do Rio de Janeiro, \\ Caixa Postal 68530, 21945-970 Rio de Janeiro, RJ, Brasil
}

Manuscript received on November 3, 2003; accepted for publication on May 19, 2004; contributed by LuIS A. MEDEIRos*

\begin{abstract}
We investigate finite approximate controllability for semilinear heat equation in noncylindrical domains. First we study the linearized problem and then by an application of the fixed point result of Leray-Schauder we obtain the finite approximate controllability for the semilinear state equation.
\end{abstract}

Key words: heat operator, finite approximate controllability, Leray-Schauder fixed point, noncylindrical.

\section{INTRODUCTION AND MAIN RESULT}

We consider a semilinear parabolic problem in a domain $\widehat{Q}$ of $\mathbb{R}^{n+1}=\mathbb{R}^{n} \times \mathbb{R}_{t}$ which is not a cylinder, but it is a union of open domains $\Omega_{t}$ of $\mathbb{R}^{n}, \quad 0<t<T$, images of a reference domain $\Omega_{0}$ by a diffeomorphism $\tau_{t}: \Omega_{0} \rightarrow \Omega_{t}$.

To make clear the notation, we identify $\Omega_{0}$ to a non empty bounded open et $\Omega$ of $\mathbb{R}^{n}$. The points of $\Omega$ are represented by $y=\left(y_{1}, y_{2}, \ldots, y_{n}\right), y_{i} \in \mathbb{R}$, for $i=1,2, \ldots, n$ and those of $\Omega_{t}$ are represented by $x=\left(x_{1}, x_{2}, \ldots, x_{n}\right), x_{i} \in \mathbb{R}, i=1,2, \ldots, n$. Thus, we have $x=\tau_{t}(y)$ or $x=\tau(y, t), \quad 0<t<T$.

\footnotetext{
*Member of Academia Brasileira de Ciências

Correspondence to: Luis A. Medeiros

E-mail: lmedeiros@abc.org.br

AMS Classification: 35B99; 35K05; 93B05.
} 
We define the noncylindrical domain $\widehat{Q}$ contained in $\mathbb{R}^{n+1}$ by

$$
\widehat{Q}=\bigcup_{0<t<T}\left\{\Omega_{t} \times\{t\}\right\} .
$$

The boundary of $\Omega_{t}$ is represented by $\Gamma_{t}$ and the lateral boundary $\widehat{\Sigma}$ of $\widehat{Q}$ is defined by

$$
\widehat{\Sigma}=\bigcup_{0<t<T}\left\{\Gamma_{t} \times\{t\}\right\} .
$$

By $Q$ we represent the cylinder

$$
Q=\Omega \times(0, T)
$$

with $\Omega$ the reference domain, with lateral boundary $\Sigma$ given by

$$
\Sigma=\Gamma \times(0, T)
$$

where $\Gamma$ is the boundary of $\Omega$.

Thus we have the diffeomorphism $\tau_{t}: Q \rightarrow \widehat{Q}$ given by

$$
(y, t) \in Q \rightarrow(x, t) \in \widehat{Q} \quad \text { with } \quad(x, t)=\left(\tau_{t}(y), t\right)=(\tau(y, t), t) .
$$

We need the following assumptions:

(A1) $\tau_{t}$ is a $C^{2}$ diffeomorphism from $\Omega$ to $\Omega_{t}$, for all $0<t<T$.

(A2) $\tau(y, t) \in C^{0}\left(0, T ; C^{2}(\bar{\Omega})\right)$.

Note that $\Omega$ is a non empty bounded open set of $\mathbb{R}^{n}$ which boundary $\Gamma$ we suppose $C^{2}$.

In this article we investigate finite approximate controllability for the following semilinear parabolic system

$$
\mid \begin{aligned}
& u^{\prime}-\Delta u+f(u)=h(x, t) \chi_{\hat{q}} \text { in } \widehat{Q} \\
& u=0 \text { on } \widehat{\Sigma} \\
& u(x, 0)=u^{0}(x) \text { in } \Omega
\end{aligned}
$$

In (1.1) we denote $u=u(x, t), \quad u^{\prime}=\frac{\partial u}{\partial t}, \Delta$ is the Laplace operator in $\mathbb{R}^{n} ; \hat{q}$ is an open subset contained in $\widehat{Q}$. Note that we consider $\hat{q}$ the image by $\tau_{t}$ of a cylinder $q$ contained in $Q$, of the type $q=w \times(0, T), w \subset \Omega$. We denote by $w_{t}$ the sections of $\hat{q}$ at level $0<t<T$, $w_{t} \subset \Omega_{t}$. By $\chi_{\hat{q}}$ we represent the characteristic function of $\hat{q}$. The function $h=h(x, t)$ is the control function such that $h(x, t) \chi_{\hat{q}}$ acts on the state $u=u(x, t)$ localized in $\hat{q}$.

The non linear function $f=f(u)$ is real, globally Lipschitz and we suppose that $f(0)=0$, that is, there exists a positive constant $K_{0}$, such that

$$
|f(\xi)-f(\eta)| \leq K_{0}|\xi-\eta|
$$


for all $\xi, \eta \in \mathbb{R}$.

To formulate our problem, let $E$ be a finite dimensional subspace of $L^{2}\left(\Omega_{T}\right)$ and denote by $\pi_{E}$ the orthogonal projection from $L^{2}\left(\Omega_{T}\right)$ onto $E$.

The problem of finite approximate controllability for (1.1) can be formulate as follows: given $T>0, \quad u^{0} \in L^{2}(\Omega), \quad u^{1} \in L^{2}\left(\Omega_{T}\right)$ and $\varepsilon>0$, to find a control $h \in L^{2}(\hat{q})$ such that the corresponding solution $u=u(x, t)$ of $(1.1)$, satisfies the conditions

$$
\begin{gathered}
\left|u(T)-u^{1}\right|_{L^{2}\left(\Omega_{T}\right)}<\varepsilon \\
\pi_{E} u(T)=\pi_{E} u^{1} .
\end{gathered}
$$

This means that the control $h=h(x, t)$ can be chosen such that $u(T)$ satisfies (1.3) and simultaneously a finite number of constrains, that is the condition (1.4).

There is an extensive literature about finite-approximate controllability for linear and semilinear heat equation in cylindrical domains. Among these works, it is worth mentioning the articles of Fernandez and Zuazua 1999, Lions 1991 and Zuazua 1997, 1999. For basic results on Sobolev spaces see Lions and Magenes 1968.

In the context of linear heat equation in noncylindrical domains, Limaco et al. 2002 proved the finite-approximate controllability. In Menezes et al. 2002 is given a proof of null controllability of the semilinear case (1.1). In Limaco et al. 2002 is mentioned some basic references on the mathematical analysis of partial differential equations in noncylindrical domains.

The main result in the present article is:

Theorem 1.1. Assume $f$ is $C^{1}$ and satisfies (1.2) with $f(0)=0$. Then, for all $T>0$, the system (1.1) is finite-approximately controllable.

This means, for any finite-dimensional subspace $E$ of $L^{2}\left(\Omega_{T}\right), \quad u^{0} \in L^{2}(\Omega), \quad u^{1} \in L^{2}\left(\Omega_{T}\right)$ and $\varepsilon>0$, there exists a control $h \in L^{2}(\hat{q})$ such that the solution $u$ of (1.1) satisfies (1.3) and (1.4), for $T>0$ given.

The methodology of the proof of the Theorem 1.1 is based in the fixed point argument, see Zuazua 1999.

There is however a new and no trivial difficulty related to the fact that $\widehat{Q}$ is noncylindrical. To set up this point we employ the idea contained in Limaco et al. 2002.

The first step in the fixed point method is to study the finite-approximate controllability for a linearized system. The application of this method is based on the unique continuation property due to Fabre 1996.

The present paper is organized as follows: Section 2 is devoted to prove the finite-approximate controllability for the linearized system. In Section 3 we prove Theorem 1.1 by a fixed point method. 


\section{ANALYSIS OF THE LINEARIZED SYSTEM}

The main result of this paper is proved in Section 3 by argument of fixed point. As an step preliminary we need to analyse the finite-approximate controllability of the following linearized system:

$$
\mid \begin{aligned}
& u^{\prime}-\Delta u+a(x, t) u=h(x, t) \text { in } \widehat{Q} \\
& u=0 \text { on } \widehat{\Sigma} \\
& u(x, 0)=u^{0}(x) \text { in } \Omega,
\end{aligned}
$$

where the potential $a=a(x, t)$ is assumed to be in $L^{\infty}(\widehat{Q})$.

As in Limaco et al. 2002 a function $u=u(x, t)$ defined in $\widehat{Q}$ is said to be strong solution for the boundary value problem (2.1), if

$$
u \in C^{0}\left([0, T], H_{0}^{1}\left(\Omega_{t}\right)\right) \cap L^{2}\left(0, T ; H^{2}\left(\Omega_{t}\right) \cap H_{0}^{1}\left(\Omega_{t}\right)\right) \cap H^{1}\left(0, T ; L^{2}\left(\Omega_{t}\right)\right)
$$

and the conditions in (2.1) are satisfied almost everywhere in their corresponding domains.

We say that a function $u=u(x, t)$ is a weak solution of $(2.1)$ if

$$
u \in C^{0}\left([0, T] ; L^{2}\left(\Omega_{t}\right)\right) \cap L^{2}\left(0, T ; H_{0}^{1}\left(\Omega_{t}\right)\right)
$$

and

$$
\begin{gathered}
\int_{0}^{T} \int_{\Omega_{t}} u \varphi^{\prime} d x d t+\int_{\Omega} u^{0}(x) \varphi(x, 0) d x+ \\
+\int_{0}^{T} \int_{\Omega_{t}} a(x, t) u \varphi d x d t+\int_{0}^{T} \int_{\Omega_{t}} \nabla_{x} u \cdot \nabla_{x} \varphi d x d t=\int_{0}^{T} \int_{\Omega_{t}} h \varphi d x d t
\end{gathered}
$$

for all $\varphi \in L^{2}\left(0, T ; H^{1}\left(\Omega_{t}\right)\right) \cap C^{1}\left([0, T] ; L^{2}\left(\Omega_{t}\right)\right)$ such that $\varphi(T)=0$.

Theorem 2.1. (Existence of Strong and Weak Solution).

a) If $u^{0} \in H_{0}^{1}(\Omega), \quad a(x, t) \in L^{\infty}(\widehat{Q})$ and $h \in L^{2}\left(0, T ; L^{2}\left(\Omega_{t}\right)\right)$, the problem (2.1) has a unique strong solution.

b) Given $u^{0} \in L^{2}(\Omega), \quad a(x, t) \in L^{\infty}(\widehat{Q})$ and $h \in L^{2}\left(0, T ; H^{-1}\left(\Omega_{t}\right)\right)$, there exists a unique weak solution of (2.1).

Proof. See Menezes et al. 2002 where the authors employ the argument consisting in transforming the heat equation in the noncylindrical domain $\widehat{Q}$ into a variable coefficients parabolic equation in a reference cylinder $Q$, by means of the diffeomorphism

$$
(x, t)=\left(\tau_{t}(y), t\right)=(\tau(y, t), t) \quad \text { for } \quad x \in \Omega_{t}, y \in \Omega
$$

and $0 \leq t \leq T$, that is, for $(x, t) \in \widehat{Q}$ and $(y, t) \in Q$. 
In fact, set

$$
v(y, t)=u\left(\tau_{t}(y), t\right)=u(\tau(y, t), t) \text { for } y \in \Omega \text { and } 0 \leq t \leq T .
$$

Equivalently

$$
u(x, t)=v\left(\tau_{t}^{-1}(x), t\right)=v(\rho(x, t), t) \text { for } x \in \Omega_{t} \text { and } 0 \leq t \leq T,
$$

where $\tau_{t}^{-1}$ denotes the inverse of $\tau_{t}$, which, according to (A1) is a $C^{2}$ application from $\Omega_{t}$ to $\Omega$ for $0 \leq t \leq T$ which we denote by $\rho_{t}$. We shall also employ the notation $\rho(x, t)=\rho_{t}(x)$, $y_{j}=\rho_{j}(x, t), \quad 1 \leq j \leq n$.

It follows that $u=u(x, t)$ solves (2.1) if and only if $v=v(y, t)$ satisfies:

$$
\mid \begin{aligned}
& \frac{\partial v}{\partial t}-\sum_{i=1}^{n} \frac{\partial^{2} v}{\partial y_{j} \partial y_{k}} \frac{\partial \rho_{i}}{\partial x_{i}} \frac{\partial \rho_{k}}{\partial x_{i}}-\sum_{i=1}^{n} \frac{\partial v}{\partial y_{j}} \Delta \rho_{j}+ \\
& \quad+\tilde{b} \cdot \nabla_{y} v+a(y, t) v=h(y, t) \text { in } Q \\
& v=0 \text { on } \Sigma \\
& v(y, 0)=u^{0}(y) \text { in } \Omega .
\end{aligned}
$$

In (2.7) we have $\tilde{b}=\tilde{b}(x, t)=\frac{\partial \rho(x, t)}{\partial t} \cdot$ Note that according to (A1) we have

$$
\tilde{b} \in C^{1}(\bar{\Omega})
$$

ANALYSIS OF THE OpERATOR. $A(t) v=-\sum_{i=1}^{n} \frac{\partial^{2} v}{\partial y_{j} \partial y_{k}} \frac{\partial \rho_{i}}{\partial x_{i}} \frac{\partial \rho_{k}}{\partial x_{i}}$

For $v, \varphi \in L^{2}\left(0, T ; H_{0}^{1}(\Omega)\right)$ and Gauss' Lemma we obtain the bilinear form $a(t, v, \varphi)$ defined by

$$
a(t, v, \varphi)=(A(t) v, \varphi)=\sum_{i=1}^{n} \int_{\Omega} \frac{\partial v}{\partial y_{j}} \frac{\partial \varphi}{\partial y_{k}} \frac{\partial \rho_{i}}{\partial x_{i}} \frac{\partial \rho_{k}}{\partial x_{i}} d x
$$

This bilinear form is bounded and $H_{0}^{1}(\Omega)$-coercive.

In (2.7) set

$$
b(y, t)=\tilde{b}(y, t)-\Delta \rho(y, t), \quad \text { where } \quad b \in\left[L^{\infty}(Q)\right]^{n} .
$$

Thus, from (2.7) the transformed of (2.1) in $Q$ is the following system

$$
\mid \begin{aligned}
& v^{\prime}+A(t) v+a(y, t) v+b \cdot \nabla v=h(y, t) \text { in } Q \\
& v=0 \text { on } \Sigma \\
& v(y, 0)=u^{0}(y) \text { in } \Omega .
\end{aligned}
$$


Note that (2.10) is a linear parabolic system with variable coefficients in a cylinder $Q=$ $\Omega \times(0, T), \quad \Omega$ a regular bounded non empty open set of $\mathbb{R}^{n}$. Since $A(t)$ is coercive, the initial boundary value problem (2.10) is a classical problem investigated in Lions and Magenes 1968.

It follows that if we take $u^{0} \in H_{0}^{1}(\Omega)$ (resp. $\left.u^{0} \in L^{2}(\Omega)\right)$ and $h \in L^{2}\left(0, T ; L^{2}(\Omega)\right)$ (resp. $h \in$ $L^{2}\left(0, T ; H^{-1}(\Omega)\right)$, then $(2.10)$ has a unique strong (resp. weak) solution $v \in C^{0}\left([0, T] ; H_{0}^{1}(\Omega)\right) \cap$ $L^{2}\left(0, T ; H^{2}(\Omega)\right) \cap H^{1}\left(0, T ; L^{2}(\Omega)\right)\left(\right.$ resp. $v \in C^{0}\left([0, T] ; L^{2}(\Omega)\right) \cap L^{2}\left(0, T ; H_{0}^{1}(\Omega)\right)$ ).

By means of the change of variable $y \rightarrow x$ we deduce the existence of a unique strong (resp. weak) solution $u$ for the system (2.1).

At this point we underline that, under assumptions (A1) and (A2), the transformation $y \rightarrow x$ does indeed, map the space of functions given in (2.2) (resp. (2.3)) into the space

$$
C^{0}\left([0, T] ; H_{0}^{1}(\Omega)\right) \cap L^{2}\left(0, T ; L^{2}\left(\Omega_{t}\right)\right) \cap H^{1}\left(0, T ; L^{2}(\Omega)\right)
$$

(resp. $\left.C^{0}\left([0, T] ; L^{2}\left(\Omega_{t}\right)\right) \cap L^{2}\left(0, T ; H_{0}^{1}\left(\Omega_{t}\right)\right)\right)$.

We have the following result

THEOREM 2.2. The system (2.1) is finite-approximate controllable. More precisely, for any $T>0$, $u^{0} \in L^{2}(\Omega), \quad u^{1} \in L^{2}\left(\Omega_{T}\right), \quad \varepsilon>0$ and $E$, finite dimensional subspace of $L^{2}\left(\Omega_{T}\right)$, there exists a control $h \in L^{2}(\hat{q})$ such that the corresponding solution of (2.1) satisfies

$$
\begin{gathered}
\left|u(T)-u^{1}\right|_{L^{2}\left(\Omega_{T}\right)}<\varepsilon \\
\pi_{E} u(T)=\pi_{E} u^{1} .
\end{gathered}
$$

Moreover, for any $R>0$ there exists a constant $C(R)>0$ such that

$$
|h|_{L^{2}(\hat{q})} \leq C(R)
$$

for any $a \in L^{\infty}(\widehat{Q})$ satisfying

$$
|a|_{L^{\infty}(\widehat{Q})} \leq R
$$

REMARK 2.1. Theorem 2.2 does not provide any estimation how the norm of the control $h$ depends $\Sigma, u^{0}, u^{1}$ and $\varepsilon>0$. However, (2.13) guarantees that $h$ remains uniformly bounded when the potential a remains bounded in $L^{\infty}$.

The control $h$ is not unique. The method we develop below provides the control of minimal $L^{2}$-norm. It is this control of minimal norm which satisfies the uniform boundness condition (2.13).

Proof of Theorem 2.2. To prove it we employ exactly the same argument as in Limaco et al. 2002. Without loss of generality we may assume $u^{0}=0$. Indeed, otherwise, we consider the 
solution $z$ of

$$
\mid \begin{aligned}
& z^{\prime}-\Delta z+a z=0 \text { in } \widehat{Q} \\
& z=0 \text { on } \Sigma \\
& z(x, 0)=u^{0} \text { in } \Omega .
\end{aligned}
$$

Then, the solution $u$ of (2.1) may be decomposed as $u=w+z$ where $w$ solves

$$
\mid \begin{aligned}
& w^{\prime}-\Delta w+a w=h \chi_{\hat{q}} \text { in } \widehat{Q} \\
& w=0 \text { on } \widehat{\Sigma} \\
& w(x, 0)=0 \text { in } \Omega .
\end{aligned}
$$

Then (2.11)-(2.12) are equivalent to

$$
\mid \begin{aligned}
& \left|w(T)-\left[u^{1}-z(T)\right]\right|_{L^{2}\left(\Omega_{T}\right)}<\varepsilon \\
& \pi_{E} w(T)=\pi_{E}\left[u^{1}-z(T)\right] .
\end{aligned}
$$

Therefore we will consider $u^{0}=0$. The regularizing effect of the heat equation allows to show that

$$
\begin{aligned}
& z(T) \text { remains in a relatively compact set of } L^{2}\left(\Omega_{T}\right) \\
& \text { when the potential a belongs to the class (2.14). } \\
& \text { This is important to obtain the uniform bound (2.13). }
\end{aligned}
$$

Let us consider the adjoint system

$$
\mid \begin{aligned}
& -\varphi^{\prime}-\Delta \varphi+a \varphi=0 \text { in } \widehat{Q} \\
& \varphi=0 \text { on } \widehat{\Sigma} \\
& \varphi(x, T)=\varphi^{0} \text { in } \Omega_{T} .
\end{aligned}
$$

Taking in account that the potential $a$ is bounded it follows that for any $\varphi^{0} \in L^{2}\left(\Omega_{T}\right)$, the system (2.15) has a unique solution $\varphi \in C^{0}\left([0, T] ; L^{2}\left(\Omega_{t}\right)\right) \cap L^{2}\left(0, T ; H_{0}^{1}\left(\Omega_{t}\right)\right)$.

We consider the functional $J$ defined in $L^{2}\left(\Omega_{T}\right)$ by

$$
J\left(\varphi^{0}\right)=\frac{1}{2} \int_{\hat{q}} \varphi^{2} d x d t+\varepsilon\left|\left(I-\pi_{E}\right) \varphi^{0}\right|_{L^{2}\left(\Omega_{T}\right)}-\int_{\Omega_{T}} u^{1} \varphi^{0} d x
$$

where $\varphi$ is the unique solution of (2.15) with initial data $\varphi^{0}$.

The functional $J$ is continuous and strictly convex in $L^{2}\left(\Omega_{T}\right)$. More precisely, by the argument of Limaco et al. 2002 we can prove that

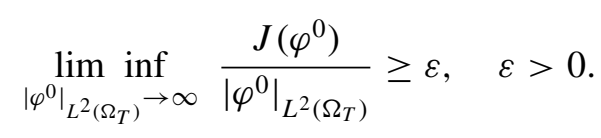


One of the key point of the proof of (2.17) is the unique continuation result of Fabre 1996.

Thus, it follows that $J$ has a unique minimizes $\hat{\varphi}^{0}$ in $L^{2}\left(\Omega_{T}\right)$. If $\hat{\varphi}$ is the solution of (2.15) associated to the minimizer $\hat{\varphi}^{0}$, then the control $h=\hat{\varphi}$ is such that the solution of (2.1) satisfies (2.11) and (2.12).

Thus it is proved the finite-approximate controllability.

In order to prove the uniform boundedness (2.13) for $h=\hat{\varphi}$, the problem may be reduced to the case $u^{0}=0$ as indicated above, provided $u^{1}$ belongs to a relatively compact set of $L^{2}\left(\Omega_{T}\right)$.

Proposition 2.3. Let $R>0$ and $K$ be a relatively compact set of $L^{2}\left(\Omega_{T}\right)$. Then, the coercivity property (2.7) holds uniformly for $u^{1} \in K$ and potential a satisfying (2.14).

Remark 2.2. Note that the functional $J$ depends on the potential $a$ and the target $u^{1}$. Thus, the Proposition 2.3 guarantees the uniform coercivity of the functional when $u^{1} \in K, K$ compact set in $L^{2}\left(\Omega_{T}\right)$ and the potential $a$ is uniformly bounded.

As a consequence of the Proposition 2.3 we deduce that the minimizers $\hat{\varphi}^{0}$ of the functional $J$ are uniformly bounded, $u^{1} \in K$ and the potential $a$ is uniformly bounded. Consequently, the controls $h=\hat{\varphi}$ are uniformly bounded.

Therefore, the proof of Theorem 2.2 is a consequence of the proof of Proposition 2.3.

Proof of Proposition 2.3. We argue by contradiction. If the coercivity property (2.17) does not hold uniformly, we deduce the existence of sequences

$$
\begin{gathered}
u_{j}^{1} \in K \\
\varphi_{j}^{0} \in L^{2}\left(\Omega_{T}\right), \quad\left|\varphi_{j}^{0}\right|_{L^{2}\left(\Omega_{T}\right)} \rightarrow \infty
\end{gathered}
$$

and

$$
\mid \begin{aligned}
& a_{j} \in L^{\infty}(\widehat{Q}) \\
& \left|a_{j}\right|_{L^{\infty}(\widehat{Q})} \leq R
\end{aligned}
$$

such that

$$
\frac{J_{j}\left(\varphi_{j}^{0}\right)}{\left|\varphi_{j}^{0}\right|_{L^{2}\left(\Omega_{T}\right)}} \leq \varepsilon-\delta
$$

for some $0<\delta<\varepsilon$.

Note that $J_{j}$ is the functional (2.16) corresponding to $u_{j}^{1}$ and $a_{j}$. Set

$$
\hat{\varphi}_{j}^{0}=\frac{\varphi_{j}^{0}}{\left|\varphi_{j}^{0}\right|_{L^{2}\left(\Omega_{T}\right)}} ; \quad \hat{\varphi}_{j}=\frac{\varphi_{j}}{\left|\varphi_{j}^{0}\right|_{L^{2}\left(\Omega_{T}\right)}}
$$


We have

$$
\begin{aligned}
\frac{J\left(\varphi_{j}^{0}\right)}{\left|\varphi_{j}^{0}\right|_{L^{2}\left(\Omega_{T}\right)}} & =\frac{\left|\varphi_{j}^{0}\right|_{L^{2}\left(\Omega_{T}\right)}}{2} \int_{\hat{q}}\left|\hat{\varphi}_{j}\right|^{2} d x d t+ \\
& +\varepsilon\left|\left(I-\pi_{E}\right) \hat{\varphi}_{j}^{0}\right|_{L^{2}\left(\Omega_{T}\right)}-\int_{\Omega_{T}} u_{j}^{1} \hat{\varphi}_{j}^{0} d x .
\end{aligned}
$$

By (2.19) and (2.21) we deduce

$$
\int_{\hat{q}}\left|\hat{\varphi}_{j}\right| d x d t \rightarrow 0 \quad \text { as } \quad j \rightarrow \infty .
$$

Extracting a sequence we also have

$$
\begin{gathered}
\hat{\varphi}_{j}^{0} \rightarrow \hat{\varphi}^{0} \quad \text { weakly in } L^{2}\left(\Omega_{T}\right) \\
\hat{\varphi}_{j} \rightarrow \hat{\varphi} \quad \text { weakly in } L^{2}\left(0, T ; H_{0}^{1}\left(\Omega_{T}\right)\right) \\
u_{j}^{1} \rightarrow u^{1} \quad \text { strongly in } L^{2}\left(\Omega_{T}\right) \\
a_{j} \rightarrow a \text { weak star in } L^{\infty}(\widehat{Q})
\end{gathered}
$$

By (2.24) we have

$$
\hat{\varphi}=0 \text { a.e. in } \hat{q}
$$

On the other hand, $\hat{\varphi}$ solves

$$
\mid \begin{aligned}
& -\hat{\varphi}^{\prime}-\Delta \hat{\varphi}+a \hat{\varphi}=0 \text { in } \widehat{Q} \\
& \hat{\varphi}=0 \text { on } \widehat{\Sigma} \\
& \hat{\varphi}(T)=\hat{\varphi}^{0} \text { in } \Omega_{T}
\end{aligned}
$$

Note that $a$ is given by (2.28) and $\hat{\varphi}^{0}$ by (2.25).

In order to obtain (2.30) we need to show that

$$
a_{j} \hat{\varphi}_{j} \rightarrow a \hat{\varphi} \quad \text { weakly in } \quad L^{2}(\widehat{Q}) .
$$

In fact, with the change of variables $x \rightarrow y$ form $\widehat{Q}$ into $Q$, the system

$$
\mid \begin{aligned}
& -\hat{\varphi}^{\prime}-\Delta \hat{\varphi}_{j}+a_{j} \hat{\varphi}_{j}=0 \text { in } \widehat{Q} \\
& \hat{\varphi}_{j}=0 \quad \text { on } \widehat{\Sigma} \\
& \hat{\varphi}_{j}(T)=\hat{\varphi}_{j}^{0} \text { in } \Omega_{T},
\end{aligned}
$$


is transformed in a new system in $Q$ with $\psi_{j}(x, t)=\varphi_{j}(y, t)$ for $y=\tau_{t}(x)$, given by

$$
\mid \begin{aligned}
& -\psi_{j}^{\prime}+A(t) \psi_{j}+a_{j} \psi_{j}+b \cdot \nabla \psi_{j}=0 \text { in } Q \\
& \psi_{j}=0 \text { on } \Sigma \\
& \psi_{j}(T)=\hat{\psi}^{0} \quad \text { in } \Omega
\end{aligned}
$$

For the parabolic problem with unknow $\psi_{j}$, in cylindrical domain $Q$, we obtain estimates which permites to apply the Aubin-Lions compactness argument for $\psi_{j}$. Then when we change variables $y \rightarrow x$ we obtain a subsequence $\left(\hat{\varphi}_{j}\right)$ in $L^{2}(\widehat{Q})$ such that

$$
\hat{\varphi}_{j} \rightarrow \hat{\varphi} \quad \text { strong } \quad L^{2}(\widehat{Q})
$$

This implies

$$
a_{j} \hat{\varphi}_{j} \rightarrow a \hat{\varphi} \quad \text { weakly in } \quad L^{2}(\widehat{Q})
$$

This justifies the conclusion that $\hat{\varphi}$ is solution of (2.30).

According to Fabre 1996 and (2.29) and (2.30) we have $\hat{\varphi}=0$ in $\widehat{Q}$. If we observe (2.25) we conclude that

$$
\hat{\varphi}_{j}^{0} \rightarrow 0 \quad \text { weakly in } \quad L^{2}\left(\Omega_{T}\right)
$$

By (2.36) and (2.27) we conclude

$$
\int_{\Omega_{T}} u_{j}^{1} \hat{\varphi}_{j}^{0} d x \rightarrow 0
$$

But, since $E$ is finity dimensional, we have

$$
\pi_{E} \hat{\varphi}_{j}^{0} \rightarrow 0 \quad \text { strongly in } \quad L^{2}\left(\Omega_{T}\right)
$$

and therefore

$$
\left|\left(I-\pi_{E}\right) \hat{\varphi}_{j}^{0}\right|_{L^{2}\left(\Omega_{T}\right)} \rightarrow 1,
$$

because $\left|\hat{\varphi}_{j}^{0}\right|_{L^{2}\left(\Omega_{T}\right)}=1$ for all $j$.

Therefore,

$$
\frac{J_{j}\left(\varphi_{j}^{0}\right)}{\left|\varphi_{j}^{0}\right|_{L^{2}\left(\Omega_{T}\right)}} \geq \varepsilon\left|\left(I-\pi_{E}\right) \hat{\varphi}_{j}^{0}\right|_{L^{2}\left(\Omega_{T}\right)}-\int_{\Omega_{T}} u_{j}^{1} \hat{\varphi}_{j}^{0} d x \rightarrow \varepsilon
$$

which is a contradiction with (2.21).

This complete the proof of Proposition 2.3 and consequently that of Theorem 2.2. 


\section{PROOF OF THE MAIN RESULT}

Now we will prove Theorem 1.1 as a consequence of Theorem 2.3 and a fixed point argument. We will begin with an existence theorem.

THEOREM 3.1. Let $f: \mathbb{R} \rightarrow \mathbb{R}$ be a $C^{1}$ function, globally Lipschitz such that $f(0)=0$. Consider $u^{0} \in L^{2}(\Omega)$ and $h \in L^{2}(\widehat{Q})$. Then, there exists only one function

$$
u \in C^{0}\left([0, T] ; L^{2}\left(\Omega_{t}\right)\right) \cap L^{2}\left(0, T ; H_{0}^{1}\left(\Omega_{t}\right)\right)
$$

solution of the problem (1.1).

Proof. We consider the change of variable (2.5) or (2.6) transforming (1.1) into

$$
\mid \begin{aligned}
& v^{\prime}+A(t) v+b \cdot \nabla_{y} v+f(v)=h(y, t), \quad \text { for } \quad(y, t) \in Q \\
& v=0 \text { for }(y, t) \in \Sigma \\
& v(y, 0)=v^{0}(y) \text { for } y \in \Omega
\end{aligned}
$$

We know that (3.1) admits a unique solution

$$
v \in C^{0}\left([0, T] ; L^{2}(\Omega)\right) \cap L^{2}\left(0, T ; H_{0}^{1}(\Omega)\right)
$$

By the change of variable $y \rightarrow x$ we deduce the existence of a unique solution $u$ of (1.1) in the class

$$
C^{0}\left([0, T] ; L^{2}\left(\Omega_{t}\right)\right) \cap L^{2}\left(0, T ; H_{0}^{1}\left(\Omega_{t}\right)\right)
$$

Proof of Theorem 1.1. As in Menezes et al. 2002 we define the function

$$
g(s)=\mid \begin{array}{ccc}
\frac{f(s)}{s} & \text { if } & s \neq 0 \\
f^{\prime}(0) & \text { if } & s=0 .
\end{array}
$$

Given $z \in L^{2}(\widehat{Q})$ let us consider the linearized system

$$
\mid \begin{aligned}
& u^{\prime}-\Delta u+g(z) u=h \chi_{\hat{q}} \text { in } \widehat{Q} \\
& u=0 \text { on } \widehat{\Sigma} \\
& u(x, 0)=u^{0}(x) \text { in } \Omega
\end{aligned}
$$

The system (3.3) is linear in $u=u(x, t)$ with potential $a=f(z) \in L^{\infty}(\widehat{Q})$ and

$$
\|a\|_{L^{\infty}(\widehat{Q})} \leq\left\|f^{\prime}\right\|_{L^{\infty}(\mathbb{R})} .
$$

Thus, will be rewritten (3.3) as

$$
\mid \begin{aligned}
& u^{\prime}-\Delta u+a u=h \chi_{\hat{q}} \text { in } \widehat{Q} \\
& u=0 \text { on } \widehat{\Sigma} \\
& u(x, 0)=u^{0}(x) \text { in } \Omega .
\end{aligned}
$$


By Theorem 2.2, for each $z \in L^{2}(\widehat{Q})$ we find a control $h=h(x, t) \in L^{2}(\hat{q})$ such that the solution $u$ of (3.5) satisfies (1.3) and (1.4). Moreover, for every $R>0$ and all potential $a=a(x, t) \in L^{\infty}(Q)$ such that $|a|_{L^{\infty}(\widehat{Q})} \leq R$ we have

$$
|h|_{L^{2}(\hat{q})} \leq C
$$

Now we define the nonlinear mapping $N$

$$
N: L^{2}(\widehat{Q}) \rightarrow L^{2}(\widehat{Q}), \quad N(z)=u
$$

The problem is reduced to finding a fixed point for $N$. Indeed, if $z \in L^{2}(\widehat{Q})$ is such that $N(z)=u=z, \quad u$ solution of (3.3) is actually solution of (1.1). Then the control $h=h(x, t)$ is that one we were looking for, since, by construction, $u=u(z)$ satisfies (1.3) and (1.4).

The nonlinear map $N: L^{2}(\widehat{Q}) \rightarrow L^{2}(\widehat{Q})$ satisfies the properties:

$$
N \text { is continuous and compact }
$$

$$
\begin{aligned}
& \text { The range of } N \text { is bounded, i.e., there exists } M>0 \\
& \text { such that }|N(z)|_{L^{2}(\widehat{Q})} \leq M \text { for all } z \in L^{2}(\widehat{Q}) .
\end{aligned}
$$

The proof of (3.8) and (3.9) is given in Menezes et al. 2002.

It follows from (3.8), (3.9) and Leray-Schauder's fixed point theorem, the mapping $N$ has a fixed point. This completes the proof of Theorem 1.1 .

\section{ACKNOWLEDGMENTS}

We take this opportunity to express our appreciation to Enrique Zuazua for stimulating conversation about the subject and for constructive remarks.

\section{RESUMO}

Este artigo é dedicado ao estudo da controlabilidade finito-aproximada para a equação não linear de transferência de calor em domínios com fronteira móvel. A demonstração do resultado principal baseia-se no princípio de continuação única de Carolina Fabre 1996 e em argumentos de ponto fixo do tipo LeraySchauder.

Palavras-chave: transferência de calor, controlabilidade aproximada finita, ponto fixo de Leray-Schauder, não cilíndrico.

\section{REFERENCES}

FABRE C. 1996. Uniqueness results for Stokes equations and their consequences in linear and nonlinear control problems, ESAIM: COCV: 267-307. 
Fernandez LA And Zuazua E. 1999. Approximate Controllability for Semilinear Heat Equation Involving Gradient Terms. J Opt Theory and Appl 101: 307-328.

LiONS J-L. 1991. Remarques sur la controlabilité approché, in Jornadas Hispano Francesas sobre controle de Sistemas Distribuidos - Univ. Malaga - Espanha: 77-87.

Lions J-L and Magenes E. 1968. Problèmes aux limites non homogènes et applications, Vol. I, Dunod, Paris.

Limaco J, Medeiros LA and Zuazua E. 2002. Existence, uniqueness and controllability for Parabolic Equations in noncylindrical domains. Matemática Contemporânea, 23 (Part II): 49-70.

Menezes SB, Limaco J and Medeiros LA. 2002. Remarks on null controllability for heat equations in thin domains. Compt and Appl Math 21: 47-65.

Zuazua E. 1997. Finite dimensional controllability for semiliner heat equations. J Math Pure Appl 76: 237-264.

ZuAZUA E. 1999. Approximate controllability for semilinear heat equation with globally Lipschitz nonlinearity, Control and Cybernetics 28: 665-683. 